\title{
CAUSES OF MORTALITY IN WILD CAPTIVE RUMINANTS OF MAHARAJBAG ZOO, NAGPUR
}

\author{
V.M. Dhoot ${ }^{1}$ and S.V. Upadhye ${ }^{2}$ \\ ${ }^{1}$ Officer-in-Charge, Maharajbag Zoo, ${ }^{2}$ Assistant Professor \\ (Veterinary Science), College of Agriculture, Nagpur, Maharashtra, \\ India.
}

For effective control and prevention of mortality in captive wild animals, it is important to be knowledgeable of various diseases affecting them. This paper presents some causes of mortality in three species of captive wild ruminants, viz., Chital (Axis axis), Nilgai (Boselaphus tragocamelus) and Sambar (Cervus unicolor) at the Maharajbag Zoo, Nagpur.

The present report is based on post-mortem findings of zoo animals that died between the year 1993 to March 2000. The species affected and the causes of death are given in Table 1.

The most common cause of mortality in Chital and Nilgai was found to be hemorrhagic shock due to traumatic injuries (8). Chital and Nilgai (1 each) had dislocation of vertebral column and three animals were found to have wounds or several injuries. One male Chital succumbed to death due to fracture of femur and shock during shifting operation, whereas, horn injury of thorax and abdomen due to infighting during rutting season was noticed in two male Chitals. Acharjyo and Rao (1987) reported 18.59 per cent deaths in captive wild ruminants due to traumatic injuries ( 85 out of 457). The workers attributed the traumatic injuries to capture operations for management/ disposal/transfer from one enclosure to the other, inter- and intra species fighting and fighting during rutting season. Paikne et al. (1990) also opined that fractures are common during rutting season. Deaths due to traumatic injuries and shock in seven males and one female in the present investigation supports the view of the earlier workers.

Another important cause of death that was noticed was respiratory failure in two Chitals, three Nilgais and one Sambar. The cause of respiratory failure in one Chital was gangrenous pneumonia whereas another died due to drowning during floods? Three Nilgai had pneumonia resulting in respiratory failure and one Sambar had hemopericardium with bread and butter appearance, adhesions of pericardium and pleura and gangrenous pneumonia. Paikne et al. (1990) reported respiratory failure in eight Chitals. Workers opined that stress due to

environmental fluctuations might be responsible for mortalities in these animals especially the young ones. Acharjyo and Rao (1987) reported 76 deaths due to pneumonia, out of a total of 457 deaths. Maximum exposure of ruminants to extreme weather as compared to other animals in captivity could be an important factor for respiratory infections in these species.

Froathy bloat and tympanitis were responsible for deaths in one Chital and Nilgai. Acute distension of rumen and reticulum due to gases results in pressure over the diaphragm and lungs leading to respiratory distress dyspnoea and death. Acharjyo and Rao (1987) have also reported deaths in two Muntjacks and one Musk Deer due to tympanitis.

Pasteurellosis caused by Pasteurella multocida, a bacterium is generally responsible for epidemics among farm and wild animals. A case of pasteurellosis was observed in a male deer. Patihar (1978-79) and Acharjyo and Rao (1987) also reported pasteurellosis in deer and Gaur respectively. Necrotic hepatitis was seen in an old female Chital. Acharjyo and Rao (1987) also observed hepatitis in six Chitals, four Muntjacks, three Nilgai, three Blackbucks and one Chousingha. In the present investigation, death due to verminous gastroenteritis was recorded in a female Nilgai. Acharjyo and Rao (1987) reported 15 deaths of captive wild ruminants due to gastroenteritis. Verminous gastroenteritis in wild ruminants seem to be important as these species are confined to the same habitat and chances of food contamination with fecal matter are always high. Similarly, identification of exact positive cases is difficult and proper deworming of these species is difficult. Mass deworming is required in which the exact dosing of proper medicine is not always possible.

\section{References}

Acharjyo, L.N. and A.T. Rao (1987). Mortality pattern in some Indian captive wild ruminants. Indian Journal of Animal Science 57(5): 430-435.

Paikne, D.L., V.P. Pathak, A.G. Ganorkar, M.R. Joshi and N.P. Dakshinkar (1990). Mortality in Zoo animals. P.K.V. Research Journal 14(10): 90-91

Parihar, N.S. (1978-79). Some pathological conditions observed in wild animals. Indian Journal of Veterinary Pathology 3: 41-42.

Table 1. Causes of mortality and the number of affected animals in different species

\begin{tabular}{llll}
\hline Cause of mortality & Chital & Nilgai & Sambar \\
\hline Haemorrhagic shock due to & & & \\
traumatic injuries & 6 & 2 & - \\
Respiratory failure & 2 & 3 & 1 \\
Frothy bloat/ tympanitis & 1 & 1 & - \\
Pasteurellosis & 1 & - & - \\
Necrotic hepatitis & 1 & - & - \\
Gastroenteritis & - & 1 & - \\
Senility & - & 1 & - \\
Total & 11 & 8 & 1 \\
\hline
\end{tabular}

Accepted for publication on 1 September 2001 